\title{
A Triangular Double Layer Microchannel Heat Sink: Effect of Parallel and Counter Flow
}

\author{
Hazli Manaf ${ }^{1 a}$, Shugata Ahmed ${ }^{1}$, Mirghani I. Ahmed ${ }^{1}$, \\ MNA Hawlader ${ }^{1}$ \\ ${ }^{1}$ Department of Mechanical Engineering, Faculty of Engineering, International Islamic University \\ Malaysia, 53100 Kuala Lumpur, Malaysia \\ aemail: hazliabmanaf@gmail.com
}

Keywords: Parallel Flow, Counter Flow, Triangular Double Layer Microchannel

\begin{abstract}
A numerical study is conducted by using ANSYS CFX 14.5, a commercial computational fluid dynamics program to predict the thermal performance of a counter and parallel flow on triangular double layered microchannel heat sink for various channel aspect ratios. Findings reveal that the counter flow configuration leads to a better heat transfer performance for low channel aspect ratio $(\alpha<4)$ and higher Reynolds Number $(\operatorname{Re}>700)$. For the parallel flow configuration, improved performance is normally shown when channel aspect ratio, $\alpha$ is more than 4 and lower Reynolds Number $(\operatorname{Re}<700)$.
\end{abstract}

\section{Introduction}

With electronic packages becoming more dense and powerful, traditional methods of thermal energy removal are reaching their limits. The removal of very large heat fluxes is becoming a hurdle to the technology roadmaps for microprocessors, power electronic modules, and many other applications incorporating microelectronic or microphotonic devices. In many of these applications, it is advantageous that the thermal management system provide high rates of heat removal uniformly over a large area [1]. One of the attractive cooling options for removing high-heat fluxes is the concept of microchannels for electronics cooling which was initially introduced by D.B. Tuckerman and R.F.W Pease [2]. This microchannel cooling concept explains that the convective heat transfer coefficient is inversely proportional with the channel width. From this concept [2], it was found that the use of high-aspect ratio to a certain level will further reduce thermal resistances and this concept has proven to be instrumental in cooling electronic devices due to inherent simplicity, reliability, low cost and has been extensively been used in recent years [2-7].

Even though the rectangular single layer micro-channel heat sink is so convenient, it is still generate a relatively high and non-uniform temperature distribution along the channel length which is lead to thermal stress in chips thus reduce the electrical performance of the packages. The high temperature gradient is attributed to the fact the large amount of heat is generated but cannot be dissipated due to an incorrect geometric dimension or shape, substrate materials or relatively low coolant's flow rate. In order to eliminate such factor, large works have been done to find out the appropriate channel's geometric parameter of rectangular single layer microchannel heat sink in order to reduce the temperature gradients either in fin thickness, channel width, channel high and etc[8-13].

Since single layer micro-channel heat sink received such attention from other researcher, there is less studies on the concept of double layer micro-channel heat sink has been done. This concept was presented by Vafai and Zhu [14] with a layer of upper channel and a layer of lower channel where the flow direction in the lower channel and upper channel are in opposite direction (counter flow). The extensively study on double layer MCHS is done by Hung et al. [15] which carried out on effects of materials, type of liquids and geometric parameters on heat transfer characteristics in a double-layer MCHS. The results of the two-layered MCHS show an improvement of average $6.3 \%$ in thermal performance over the single-layer MCHS.

The above studies on double layer MCHS investigated the counter flow configuration where the flow in the upper and lower channels are in opposing direction. A study on a parallel flow in 
double layer rectangular microchannel [16] is found and they investigated the thermal performance between the parallel and counter flow for different aspect ratio. They founded that the parallel flow configuration leads to a better heat transfer performance except for high Reynolds number and high channel aspect ratio. Since all the studies ignoring the effect of channel shape on heat transfer performance on double layer MCHS, so in the present study the thermal performance of a triangular double layer MCHS in parallel and counter flow is investigated by varies the aspect ratio and Reynolds Numbers.

\section{Physical Model}

A cross sectional view of the heat sink is given in Fig 1. The channel consists of a triangular channel and inverted triangular channel. The enlarged version of the channel is shown in Fig 1(b). The dimensions are provided in Table 1. Total footprint area of the test section is $0.5 \mathrm{~cm}^{2}$.Aluminium is considered as channel material and the investigations is carried out by using water as a coolant.

The present study investigates the heat transfer on the parallel flow (fig. 2a) and counter flow (fig. 2b) by varying geometric parameter. The arrow in the fig. 2 indicates the direction of the coolant either entering the channel or vice verse. In order to simplify the analysis, the following assumptions are made:

1. Three dimensional steady state system is accounted.

2. Gravitational effect on fluid flow is assumed to be negligible

3. Flow is incompressible, single-phase, laminar and fully developed.

4. Thermophysical properties of fluids and channel materials are considered temperature independent.

5 . Uniform heat flux of $100 \mathrm{~W} / \mathrm{cm}^{2}$ is applied at the bottom of the heat sink.

6. It is assumed that top and outer walls are adiabatic wall.

7. No heat loss by radiation.

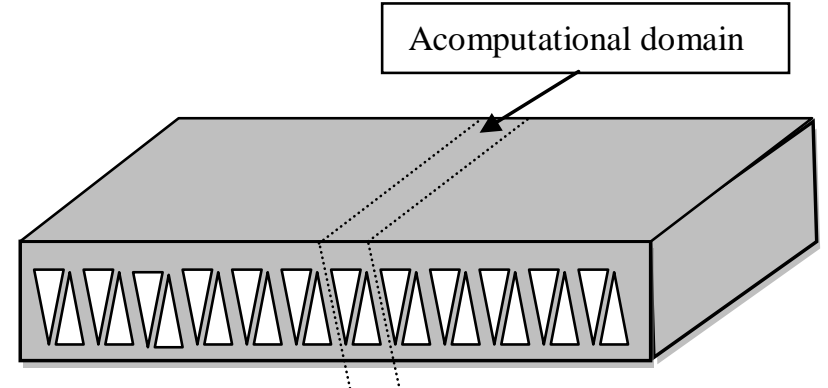

(a)

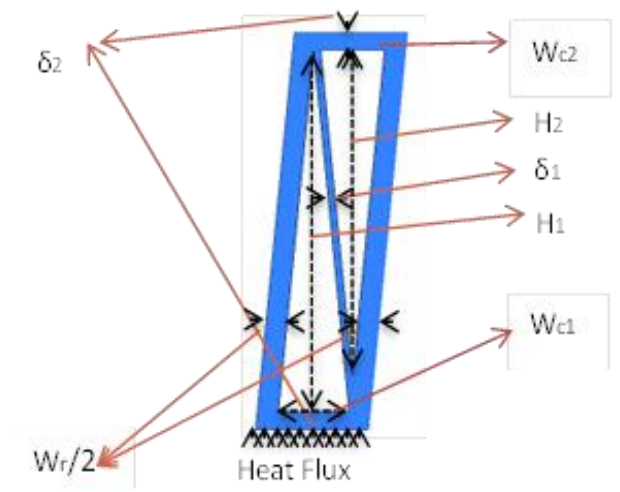

(b)

Fig. 1 Schematics diagram of Triangular Double Layer Microchannel heat sink. (a) Geometry (b) Computational domain

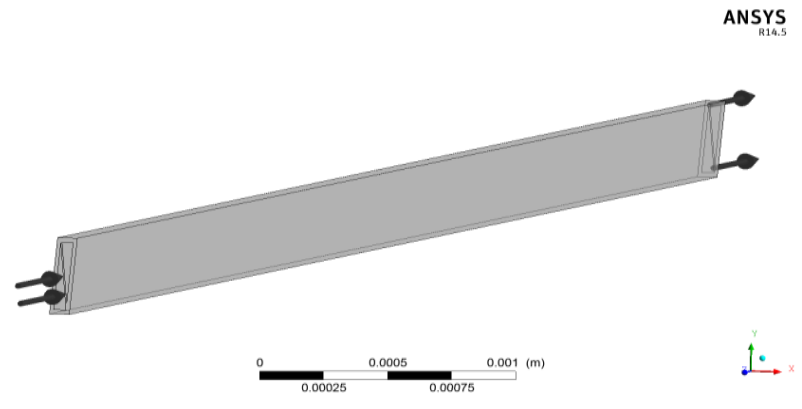

(a)

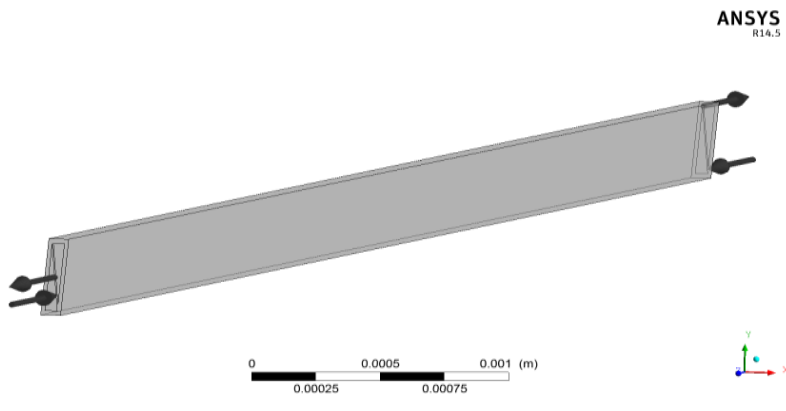

(b)

Fig. 2 Flow direction for (a) parallel flow and (b) counter flow two-layered MCHS. 
Table 1. Dimensions of the microchannel heat sink

\begin{tabular}{|l|c|}
\hline Parameter (unit) & Value Range \\
\hline Width of MCHS, $L_{x}(\mathrm{~mm})$ & 10 \\
\hline Channel Length, $L_{z}(\mathrm{~mm})$ & 5 \\
\hline Substrate thickness, $\delta 1(\mathrm{~mm})$ & 0.005 \\
\hline Horizontal rib, $\delta 2(\mathrm{~mm})$ & 0.02 \\
\hline Channel Width Ratio, $\beta=W_{c} /\left(W_{c}+W_{r}\right)$ & 0.77 \\
\hline Aspect ratio, $\alpha_{1}=H / W_{c}$ & $1-14$ \\
\hline
\end{tabular}

\section{Mathematical Model}

\subsection{Governing equations}

Three dimensional continuity equation, conservation of momentum equations and conservation of energy equations are solved numerically to obtain pressure, temperature and velocity distributions, which are given below [15]:

\section{Continuity equation:}

$\frac{\partial u}{\partial x}+\frac{\partial v}{\partial y}+\frac{\partial w}{\partial z}=0$

\section{Conservation of momentum equations:}

In the X-direction:

$\rho\left(u \frac{\partial u}{\partial x}+v \frac{\partial u}{\partial y}+w \frac{\partial u}{\partial z}\right)=-\frac{\partial P}{\partial x}+\mu\left(\frac{\partial^{2} u}{\partial x^{2}}+\frac{\partial^{2} u}{\partial y^{2}}+\frac{\partial^{2} u}{\partial z^{2}}\right)$

In the Y-direction:

$\rho\left(u \frac{\partial v}{\partial x}+v \frac{\partial v}{\partial y}+w \frac{\partial v}{\partial z}\right)=-\frac{\partial P}{\partial y}+\mu\left(\frac{\partial^{2} v}{\partial x^{2}}+\frac{\partial^{2} v}{\partial y^{2}}+\frac{\partial^{2} v}{\partial z^{2}}\right)$

In the Z-direction:

$\rho\left(u \frac{\partial w}{\partial x}+v \frac{\partial w}{\partial y}+w \frac{\partial w}{\partial z}\right)=-\frac{\partial P}{\partial z}+\mu\left(\frac{\partial^{2} w}{\partial x^{2}}+\frac{\partial^{2} w}{\partial y^{2}}+\frac{\partial^{2} w}{\partial z^{2}}\right)$

Conservation of energy equations:

Fluid domain:

$\frac{\partial^{2} T_{f}}{\partial x^{2}}+\frac{\partial^{2} T_{f}}{\partial y^{2}}+\frac{\partial^{2} T_{f}}{\partial z^{2}}=\frac{1}{\Omega}\left(u \frac{\partial T_{f}}{\partial x}+v \frac{\partial T_{f}}{\partial y}+w \frac{\partial T_{f}}{\partial z}\right)$

Solid domain:

$k_{S}\left(\frac{\partial^{2} T_{S}}{\partial x^{2}}+\frac{\partial^{2} T_{s}}{\partial y^{2}}+\frac{\partial^{2} T_{s}}{\partial z^{2}}\right)=0$

\subsection{Boundary conditions}

Following conditions are applied at the boundaries of the computational domain:

Inlet:

$T_{f}=T_{i n}, u=u_{i n}, v=w=0$

Outlet:

$P=P_{\text {out }}$

Solid-fluid interface:

A laminar sub-layer is considered at the solid-fluid interface.

$u=v=w=0, T_{f}=T_{s},-k_{f} \frac{\partial T_{f}}{\partial n}=-k_{s} \frac{\partial T_{s}}{\partial n}$

Channel bottom wall:

$q_{\text {in }=-k_{S}} \frac{\partial T_{S}}{\partial n}$

Other channel walls

$\frac{\partial T_{s}}{\partial n}=0$ 
To measure the thermal performance of the system, the concept of the total thermal resistance is applied.

$R_{t h}=\frac{T_{\max }-T_{\text {in }}}{q_{\text {in }}}$

Where, $T_{\max }$ is the highest temperature in the solid domain and $T_{\text {in }}$ is the fluid inlet temperature, and $q_{\text {in }}$ is the applied heat flux.

\section{Results and Discussion}

To check the adequacy of the simulation scheme, the predicted results is validated against experimental data of Tuckerman and Pease [2] and simulation data by Tc-Hyung [15] using the same dimensions and operating conditions. Under same channel dimensions and operating conditions, the present predictions agree with the experimental data with a average maximum difference of $1.0 \%$ in thermal resistance (Table 2).

Table 2: Comparison of the Rth for various channel dimensions and operating conditions

\begin{tabular}{|c|c|c|c|c|c|c|c|c|}
\hline $\operatorname{Exp}$ & $\begin{array}{c}\mathbf{W c} \\
(\boldsymbol{\mu \mathbf { m }})\end{array}$ & $\begin{array}{c}\mathbf{W r} \\
(\boldsymbol{\mu \mathbf { m }})\end{array}$ & $\begin{array}{c}\mathbf{H} \\
(\boldsymbol{\mu \mathbf { m }})\end{array}$ & $\mathbf{f}\left(\mathbf{c m}^{\mathbf{3}} / \mathbf{s}\right)$ & $\begin{array}{c}\text { Rth } \\
(\mathbf{K} / \mathbf{W})[\mathbf{1}]\end{array}$ & $\begin{array}{c}\text { Rth}(\mathbf{K} / \\
\mathbf{W})[\mathbf{2 5}]\end{array}$ & $\begin{array}{c}\text { Rth } \\
(\mathbf{p r e s e n t})\end{array}$ & $\begin{array}{c}\mathbf{\mathbf { Q }} \\
\left(\mathbf{W} / \mathbf{c m}^{\mathbf{2}}\right)\end{array}$ \\
\hline $\mathbf{1}$ & 56 & 44 & 320 & 4.7 & 0.110 & 0.110 & 0.109 & 181 \\
\hline $\mathbf{2}$ & 55 & 45 & 287 & 6.5 & 0.113 & 0.109 & 0.112 & 277 \\
\hline
\end{tabular}

The thermal performance of a parallel and counter flow for triangular double layer MCHS have been investigated for various values of channel aspect ratios and for Reynolds numbers between the ranges of 150 to 2300 . The Reynolds number is defined as follow:

$$
R e=\rho u_{\text {in }} D_{h} / \mu
$$

Where $\mathrm{D} \mathrm{h}$ is the hydraulic diameter which is expressed as:

$$
D_{h}=\frac{2 W_{c h} H_{c h}}{W_{c h}+\sqrt{W_{c h}^{2}+4 H_{c h}^{2}}}
$$

By set the inlet in terms of mass flow rate, the analysis on the effect of aspect ratio for any Reynolds Number was investigated for any flow arrangement. Note that, the ribs thickness, $\delta_{1}$ and horizontal ribs, $\delta_{2}$ is fixed, so that the effect of aspect ratio thoroughly investigated.

The result in fig. 3 a shows that, the total thermal resistance, Rth decreases as channel aspect ratio increases for both parallel and counter flow arrangements just before aspect ratio 6 . After that, total thermal resistance, Rth starting to increase slowly when aspect ratio increase. The explanation of this phenomenon is that, as the channel aspect ratio increase, the effective convective heat transfer area is increases thus reduce convective thermal resistance. The decreasing trend stop at aspect ratio 6 , since when further increasing the aspect ratio conductive thermal resistance will increase thus overshadow the decrement of convective thermal resistance. The result was proved by Hung et al. [15] which demonstrated that the increase in aspect ratio reduces the effective thermal resistance for the range of aspect ratios investigated in the present study.

It observed in Fig. 3a that in general the triangular double layer MCHS with parallel flow configuration gives lower effective thermal resistance as compared to counter flow except for low channel aspect ratio $(\alpha<4)$ at which the counter flow show a slightly better thermal performance. The results for the parallel flow configuration gives lower thermal resistance as compared to counter flow low channel aspect ratio $(\alpha>4)$ for the range of Reynolds number investigated. It is also observed in fig. $3 \mathrm{~b}$ that in general the triangular double layer MCHS with counter flow configuration gives lower total thermal resistance as compared to parallel flow except for low Reynolds number $(\operatorname{Re}<700)$ which the parallel flow show slightly better thermal performance. 


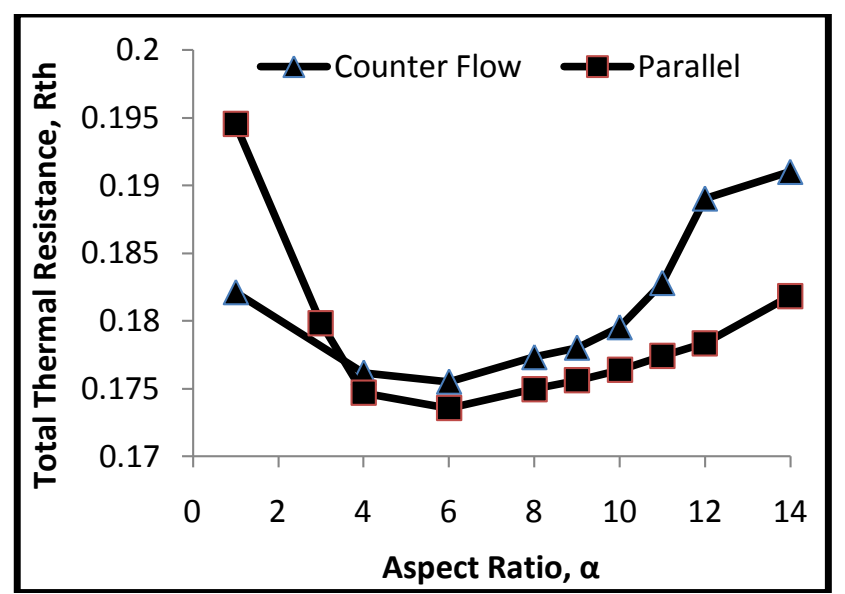

(a)

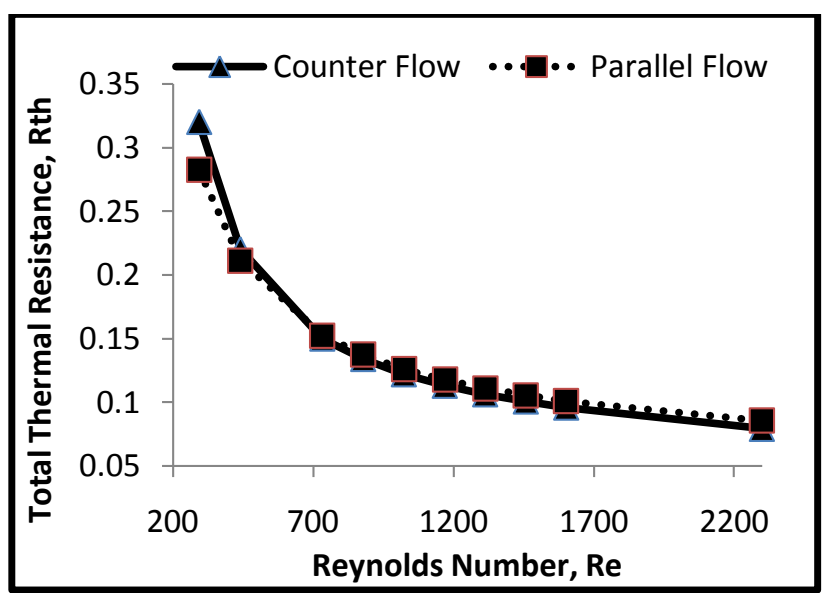

(b)

Fig. 3(a) Total Thermal Resistance verses Aspect Ratio, 3(b) Total Thermal Resistance verses Reynolds Number

However, the decrement of overall thermal resistance for parallel and counter flow arrangement is slightly different for various channel aspect ratio and various Reynolds number. From the fig. $3 \mathrm{a}$ the average total thermal resistance, $\mathrm{R}_{\mathrm{th}}$ for counter flow is $1.4 \%$ higher compare to the parallel flow arrangement. Besides that, counter flow arrangement also create a hot spot (fig.4) at the centre of the heat sink which might producing thermal stress in chips thus reduce the electrical performance of the packages via thermal breakdown,or electrical-thermal instability. For the parallel flow (fig.4), a high temperature occurs at the coolant outlet of the sinks since wall and fluid temperature difference at the outlet is small. Hence, heat transfer rate is poor near channel exit, which gives rise to surface temperature.

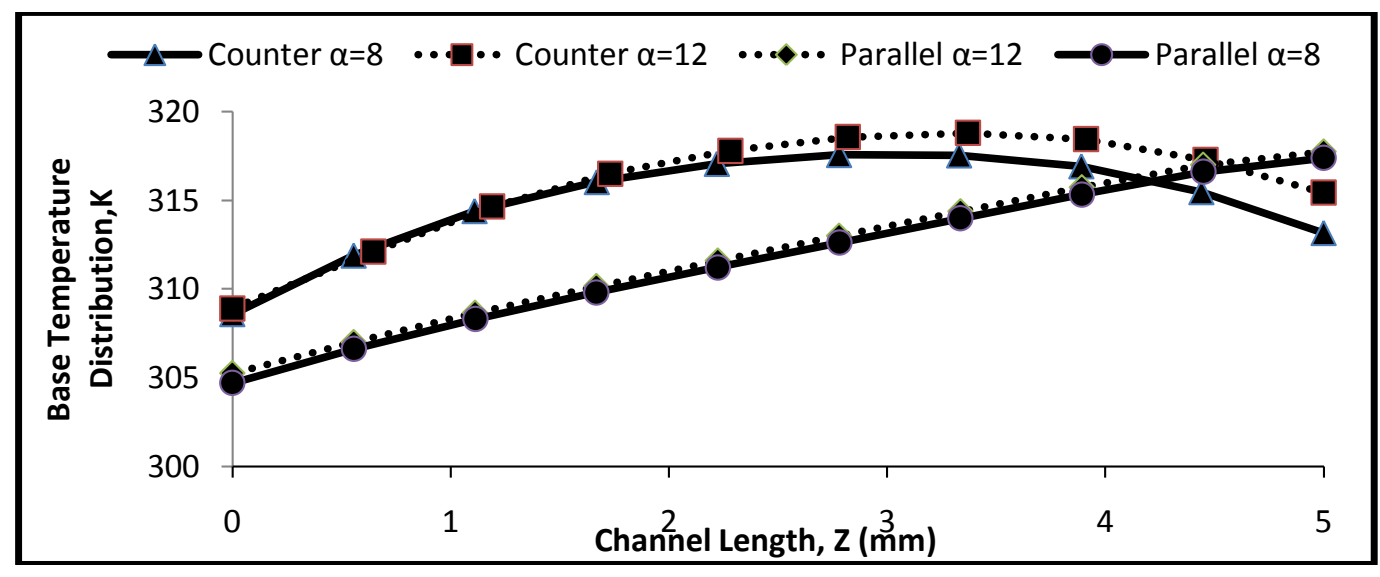

Fig 4. Heat Sink Base Temperature Distribution along the microchannel length for triangular double layer MCHS in parallel and counter flow arrangement for different aspect ratio

\section{Conclusion}

The heat transfer characteristics of Triangular MCHS for 2 type of flow arrangement are numerically analysed in details. The effect of various channel aspect ratio and Reynolds number on counter and parallel flow were studied. Based on the simulation results presented, the following conclusion can be drawn:

1. Parallel flow configuration leads to a better heat transfer performance for high channel aspect ratio $(\alpha>4$ and the counter flow configuration, the thermal performance is higher when channel aspect ratio, $\alpha$ less than 4 .

2. Counter flow configuration shows better heat transfer performance for higher Reynolds Number $(\operatorname{Re}>700)$. 


\section{Nomenclature}

$\boldsymbol{D} \boldsymbol{h} \quad$ Hydraulic Diameter $(m) \quad \boldsymbol{u}$

Hch Channel Height $(m) \quad \boldsymbol{u}_{i}$

$\boldsymbol{k}_{\boldsymbol{s}} \quad$ Solid thermal conductivity $v$

$\boldsymbol{k}_{\boldsymbol{f}} \quad$ Fluid thermal conductivity $\boldsymbol{w}$

$\begin{array}{lll}\boldsymbol{k} f & \text { Fluid thermal conductivity } & \boldsymbol{w} \\ \boldsymbol{L} z & \text { Microchannel Length }(m) & \boldsymbol{W} \boldsymbol{c h}\end{array}$

$\boldsymbol{L} \boldsymbol{x} \quad$ Microchannel Width $(m)$

$\boldsymbol{q}_{\text {in }} \quad$ Heat flux $W m-2$

$\boldsymbol{P} \quad$ Pressure $(\mathrm{Pa})$

$\boldsymbol{R} \boldsymbol{e} \quad$ Reynolds Number

$\boldsymbol{R}_{\boldsymbol{t h}} \quad$ Total thermal resistance $(K / W)$

$T_{\text {in }} \quad$ Inlet coolant temperature $(K)$

$\boldsymbol{T}_{\max } \quad$ Maximum Temperature $(K)$ $\boldsymbol{u}$

v

Greek symbols

a

$\beta$

$\boldsymbol{\mu}$

$\rho$

$d$

$\Omega$ velocity in $\mathrm{X}$ direction

Inlet velocity

Velocity in Y direction

Velocity in $\mathrm{Z}$ direction

Channel width $(m)$

Aspect ratio

Width ratio

Viscosity $(\mathrm{Pa} \mathrm{s})$

Density

Substrate thickness

Thermal diffusivity

\section{References}

1. W. Nakayama, A. E. Bergles, Cooling electronic equipment past, present and future, in: Heat Transfer in Electronic and Microelectronic Equipment, Hemisphere, New York, 1990, pp. 3-39.

2. D.B. Tuckerman, R.F.W. Pease, High-performance heat sinking for VLSI, IEEE Electronics Devices Lett. 2, 1981, pp. 126-129.

3. J.R Weilling, C.B. Wooldridge, free convection heat transfer coefficient from rectangular vertical fins, Journal of Heat Transfer 87(1965) 439-444

4. J. Li, G.P. Peterson, P. Cheng, Three-dimensional analysis of heat transfer in a micro heat sink with single phase flow, Int. J. Heat Mass Trans. 47 (2004) 4215-4231.

5. J.H. Ryu, D.H. Choi, S.J. Kim, Numerical optimization of the thermal performance of a microchannel heat sink, Int. J. Heat Mass Transfer 45, 2002, pp. 2823-2827.

6. C.W. Chen, J.J. Lee, H.S. Kou,Optimal thermal design of microchannel heat sinks by the simulated annealing method, Int. Commun. Heat Mass Transfer 35 (2008) 980-984.

7. R. Chein, J. Chen,Numerical study of the inlet/outlet arrangement effect on microchannel heat sink performance, Int. J. Therm. Sci. 48 (2009) 1627-1638.

8. S.J. Kim, Method for thermal optimization of microchannel heat sinks, Heat Transfer Eng. 25 (2004) 37-49.

9. J. Li, G.P. Peterson, Geometric optimization of a micro heat sink with liquid flow, IEEE Trans. Comp. Pack. Technol. 29 (1) (2006) 145-154.

10. H. Al-Bakhit, A. Fakheri, Numerical simulation of heat transfer in simultaneously developing flows in parallel rectangular ducts, Appl. Therm. Eng. 26 (2006) 596-603.

11. J. Li, G.P. Peterson, 3-Dimensional numerical optimization of silicon-base high performance parallel microchannel heat sink with liquid flow, Int. J. Heat Mass Transfer 50 (2007) 2895-2904.

12. T. Bello-Ochende, L. Liebenberg, J.P. Meyer, Constructal cooling channels for microchannel heat sinks, Int. J. Heat Mass Transfer 50 (2007) 4141-4150.

13. H.S. Kou, J.J. Lee, C.W. Chen, Optimum thermal performance of microchannel heat sink by adjusting channel width and height, Int. Commun. Heat Mass Transfer 35 (2008) 577-582.

14. K. Vafai, L. Zhu, Analysis of two-layered micro-channel heat sink concept in electronic cooling, Int. J. Heat Mass Transfer 31 (1999) 1176-1186.

15. T.-C. Hung, W.-M. Yan, X.-D. Wang, and Y.-X. Huang, "Optimal design of geometric parameters of double-layered microchannel heat sinks," International Journal of Heat and Mass Transfer, vol. 55, no. 11-12, pp. 3262-3272, May 2012.

16. Kok-Cheong Wong, Fashli Nazhirin Ahmad Muezzin, "Heat transfer of a parallel flow two-layered microchannel heat sink," ," International Communications in Heat and Mass Transfe, vol 49 (2013) 136-140 